\title{
Double pseudoaneurysms of the aortic bulbus after aortic valve replacement surgery
}

\author{
Stasa Dragan Krasic ${ }^{1}$, Igor Slavoljub Zivkovic ${ }^{2}$, Zorana Kovacevic ${ }^{3}$, Vladislav Vukomanovic ${ }^{4}$ \\ ${ }^{1}$ Department of Pediatrics, Mother and Child Health Care Institute of Serbia “Dr Vukan Cupic”, Belgrade, Serbia \\ ${ }^{2}$ Department of Cardiac Surgery, Dedinje Cardiovascular Institute, Belgrade, Serbia \\ ${ }^{3}$ Department of Cardiology, Dedinje Cardiovascular Institute, Belgrade, Serbia \\ ${ }^{4}$ Department of Cardiology, Mother and Child Health Care Institute of Serbia "Dr Vukan Cupic", Belgrade, Serbia; \\ School of Medicine, University of Belgrade, Belgrade, Serbia
}

Kardiochir Torakochir Pol 2019; 16 (3): 141-143

A pseudoaneurysm of the ascending aorta (PAA) represents a rare, life-threatening complication in cardiac surgery [1]. The anatomical localization and expansion lead to compression or an erosive effect on the structures of the mediastinum produces heterogeneous symptoms as well as the pulsating mass, dysphagia, hoarseness, stridor, or angina due to obstruction of the coronary blood vessel or graft. The weakness of the wall leads to an increased probability of spontaneous rupture which could result in mediastinal hemorrhage, acute cardiac tamponade, hypovolemic shock and death. Surgical treatment is mandatory because of the high risk of a fatal outcome [2].

We present a patient with double pseudoaneurysms of the ascending aorta with the origin from the right sinus which completely occluded the right coronary artery and produced ischemic heart symptoms.

A 61-year old woman was admitted with an eightmonth history of fatigue, a sense of palpitation, irritating dry cough and hoarse voice. The patient's past medical history showed that aortic valve replacement was performed 4 years ago when the mechanical prosthesis was implanted.

Transthoracic echocardiography revealed a newly formed 4-cm-wide cystic formation as well as a smaller round cavity behind it. The origin of the cyst was from the right and non-coronary sinus of Valsalva. Color Doppler showed communication between the aorta and the larger cavity during systole. Transesophageal echocardiography (TEE) revealed a cystic formation arising from the aortic root, extending in front of the right atrium and ventricle. It was $6.8 \times 4.5 \mathrm{~cm}$ in diameter, divided into two cavities, a larger front and a smaller rear with mural thrombosis.

Contrast multislice CT (MSCT) examination revealed two separated aneurysmal formations with large mural thrombosis along the right edge of the aortic bulbus, as well as anteriorly (Figures 1 A, B). The larger formation was found in the projection of the root of the right coronary ar- tery, and also the smaller pseudoaneurysm along its origin (Figure $2 \mathrm{~A}$ ).

Aortography revealed two pseudoaneurysmal formations in the right part of the aortic bulbus which completely occluded the origin of the right coronary artery, which could be visualized only due to retrograde flow from left coronary artery (Figure $2 \mathrm{~B}$ ).

The patient underwent surgery immediately after the diagnostic procedures were done. Cardiopulmonary bypass (CPB) was instituted by means of right femoral artery perfusion and right femoral vein drainage. Sternotomy was performed when the body temperature reached $25^{\circ} \mathrm{C}$ During cooling while the heart was freed from adhesions, pseudoaneurysm formation was detected from the bulbus of the aorta and origin of the right coronary artery. When the body temperature reached $19^{\circ} \mathrm{C} \mathrm{CPB}$ stopped and the aneurysm sac was opened. The aortic valve prosthesis was inspected and there was no apparent purulent material or chronic granulation tissue to suggest active endocarditis. Aortic repair was performed after debriding the pseudoaneurysm sac and trimming its edges. Patch repair was performed using a Dacron material. The transected right coronary artery was reconstructed using by $6-\mathrm{mm}$ woven graft, and a proximal anastomosis was constructed on the ascending aorta. After reconstruction CPB and the rewarming process were started. The total deep hypodermic circulatory arrest time was 35 minutes. Systemic rewarming was done and cardiopulmonary bypass was weaned off. Postoperative TEE showed an intact repair with no significant residual lesions.

There were no complications during the operative and postoperative period. The postoperative recovery was uneventful and patient was discharged home in good condition.

The available medical literature reports an incidence of less than $0.5 \%$, with a high mortality rate $(29-46 \%)[2,3]$. Previous cardiac surgery procedures increase the incidence

Address for correspondence: Igor Slavoljub Zivkovic MD, Department of Cardiac Surgery, Dedinje Cardiovascular Institute, Belgrade, Serbia, e-mail: igor88zivkovic@gmail.com

Received: 24.09.2019, accepted: 24.09.2019 

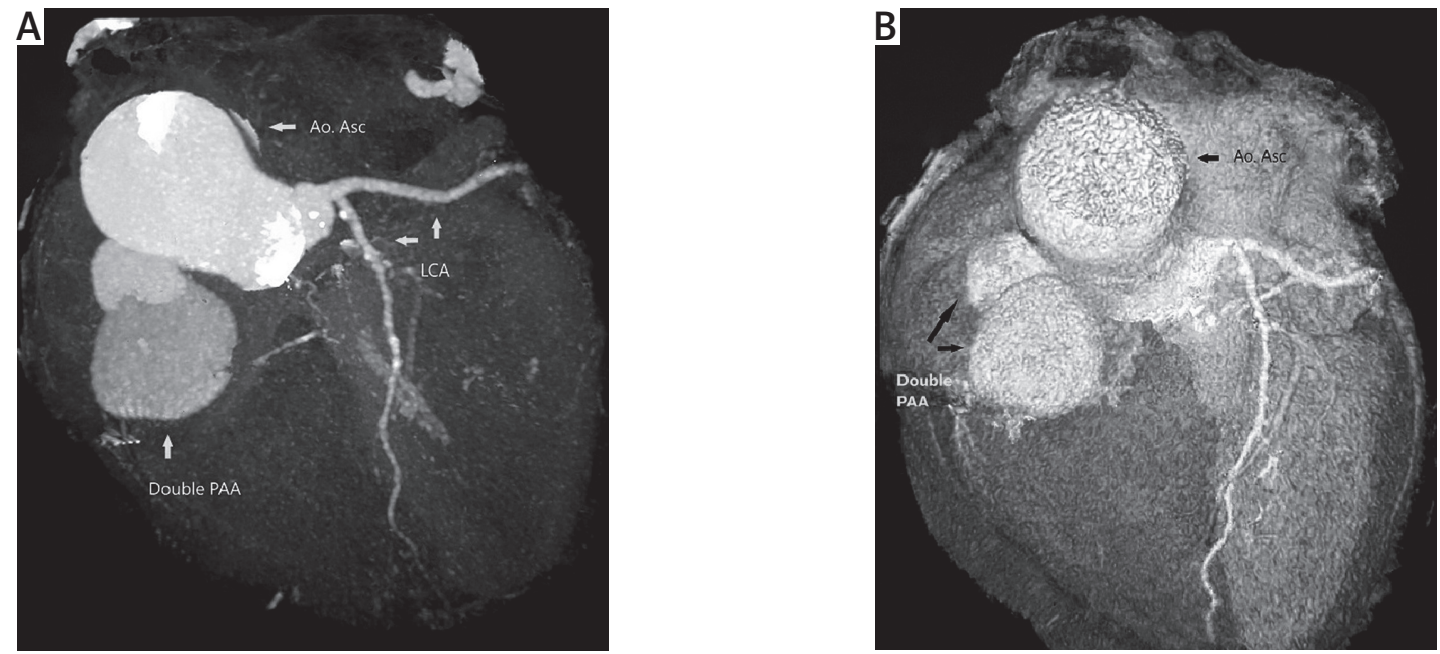

Figure 1. A - Contrast multislice CT (MSCT) examination revealed two separated aneurysmal formations with large mural thrombosis along the right edge of the aortic bulbus, as well as anteriorly. A larger formation was found in the projection of the root of the right coronary artery, and also a smaller pseudoaneurysm along its origin. B - Volume rendering reconstruction MSCT revealed two separate aneurysmal formations from the right coronary sinus with occlusion of the right coronary artery

Ao. Asc. - ascending aorta, LCA - left coronary artery, PAA - pseudoaneurysm of ascending aorta.
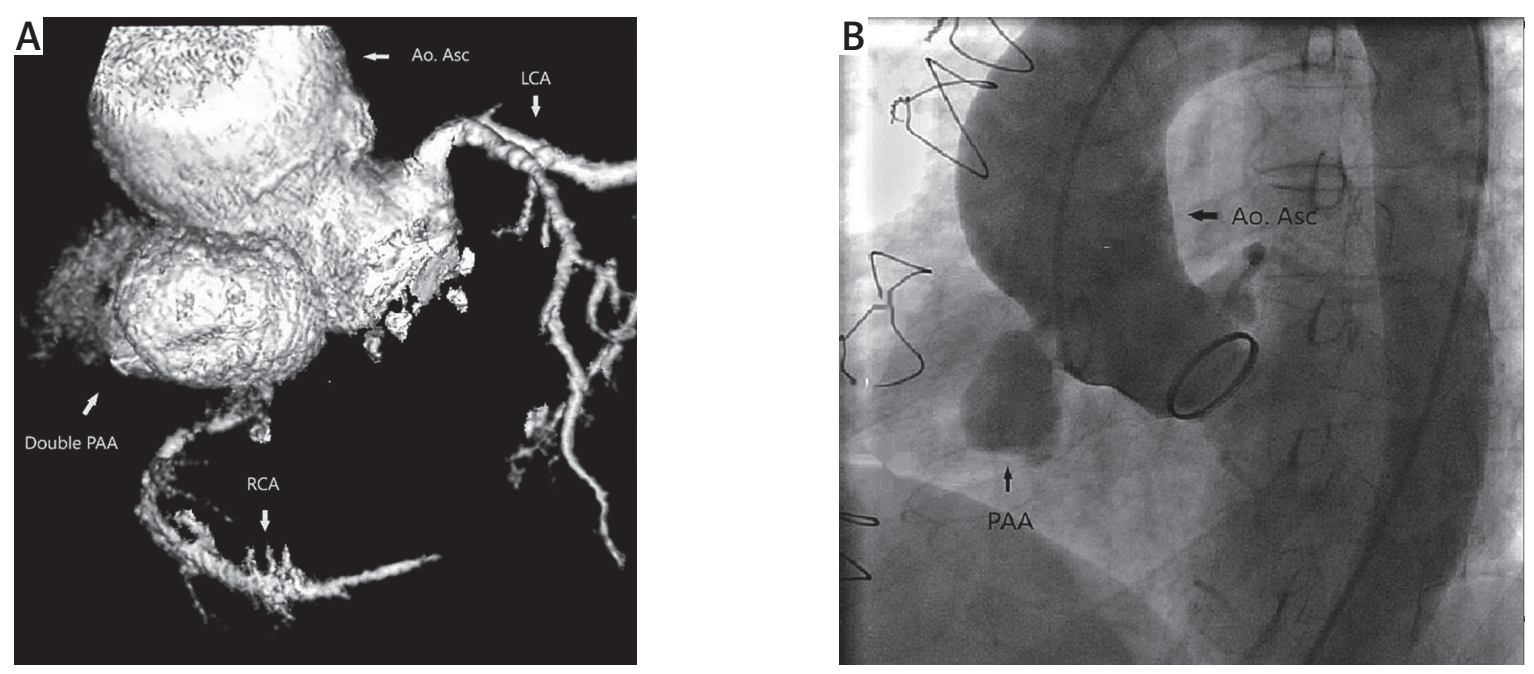

Figure 2. A - MSCT volume rendering reconstruction of arteries of the heart registered double pseudoaneurysm formation of the right coronary sinus. B - Cardiac catheterization showed occluded right coronary artery and aneurysm filled with contrast

Ao. Asc. - ascending aorta, RCA - right coronary artery, LCA - left coronary artery, PAA - pseudoaneurysm of ascending aorta.

rate more than $13 \%$. Infection, connective tissue disease, chronic hypertension, aortic calcification or trauma increase the risk for PAA developing [2]. Among those possible etiologies, infection was reported to be the most prominent cause in up to $50 \%$ of cases. Staphylococcus aureus seems to be the most isolated organisms in patients with positive blood culture [4]. The most common surgical cause of PAA is aortic injury in the area of cannulation and cross-clamp, cardioplegia needle place and on the suture lines. Approximately half of the PAA cases manifest within 2 years after surgery, but intervals up to 18 years have been reported [4]. In our case, the PAA formation developed 4 years after aortic valve replacement. The most likely place of PAA occurrence was at the previous aortotomy suture line. The cause for the PAA developing after 4 years is questionable, because our patient did not have signs of infectious endocarditis, chronic hypertension or connective tissue disease.

Cardiac catheterization and angiography used to be the gold standard in the diagnosis of pseudoaneurysm, but are no longer required due to the existence of modern non-invasive methods as well as cardiac magnetic resonance (CMR). It is, however, still necessary if the symptomatology is dominated by anginal ailments such as was the case with our patient, in whom one of the two pseudoaneurysm completely occluded the origin of the right coronary artery [2].

The surgical management of PAA remains a challenge. The literature reported a mortality rate of about $29 \%$ to $46 \%$, most frequently as a consequence of fatal hemorrhage resulting from rupture of the pseudoaneurysm dur- 
ing sternal re-entry or surgical maneuvers for its repair. Median sternotomy using a femorofemoral bypass and hypothermic circulatory arrest has been the strategy of choice [5]. Endovascular procedures are limited to patients who have very high intraoperative risk [6].

The distinctive feature of our case is the existence of a very rare double pseudoaneurysm of the aorta which occluded the origin of the right coronary artery, causing the symptoms of unstable angina pectoris. The patient was operated on and postoperative recovery was uneventful.

\section{Disclosure}

The authors report no conflict of interest.

\section{References}

1. Garisto J, Medina A, Williams D, Carrillo RG. Surgical management of giant ascending aortic pseudoaneurysm. Tex Heart Ins J 2010; 37: 710-713.

2. Parihar B, Choudhary L, Madhu AP, Alpha MK, Thankachen R, Shukla V. Pseudoaneurysm of ascending aorta after aortic valve replacement. Ann Thorac Surg 2005; 79: 705-707.

3. Jung TE, Lee DH. Surgery for pseudoaneurysm of the ascending aorta under moderate hypothermia. J Cardiothor Surg 2011; 6: 125.

4. Navaravong L, Saab F, Cook JR, Peterman M, Flack J. Ascending aortic pseudoaneurysm, a ticking bomb after cardiac surgery. Cardiovasc Revasc Med 2011; 12: 177-180.

5. Emaminia A, Amirghofran AA, Shafa M, Moaref A, Javan R. Ascending aortic pseudoaneurysm after aortic valve replacement: watch the tip of the cardioplegia cannula! J Thorac Cardiovasc Surg 2009; 137: 1285-1286.

6. Quevedo HC, Santiago-Trinidad R, Castellanos J, Atianzar K, Anwar A, Abi Rafeh N. Systematic review of interventions to repair ascending aortic pseudoaneurysms. Ochsner J 2014; 14: 576-585. 